\title{
Recruitment of African Americans With Chronic Renal Insufficiency Into a Multicenter Clinical Trial: The African American Study of Kidney Disease and Hypertension
}

\author{
Robert A. Phillips, MD, PhD; ${ }^{1}$ Marquetta Faulkner, MD; ${ }^{2}$ Jennifer Gassman, PhD; ${ }^{3}$ Luzmaria Jaen, RN; ${ }^{4}$ \\ John W. Kusek, PhD; ${ }^{5}$ Keith Norris, MD; ${ }^{6}$ Akinlolu Ojo, MD, PhD; ${ }^{7}$ \\ for the African American Study of Kidney Disease and Hypertension Study Group
}

In patients with hypertensive nephrosclerosis, the African American Study of Kidney Disease and Hypertension (AASK) demonstrated the superiority of angiotensin-converting enzyme inhibitor therapy in blunting progression of renal disease compared with a $\beta$ blocker and a dibydropyridine calcium channel blocker. In addition, the study found that a blood pressure treatment strategy that resulted in an achieved blood pressure of 128/78 mm Hg (low blood pressure goal) was no more effective in slowing the progression of renal disease than a strategy that resulted in a blood pressure of 141/85 $\mathrm{mm} \mathrm{Hg}$ (usual blood pressure goal). AASK, which enrolled only African Americans with mild to moderate chronic renal

From Lenox Hill Hospital and NYU School of Medicine, New York, NY; ${ }^{1}$ Meharry Medical College, Nashville, TN; ${ }^{2}$ The Cleveland Clinic Foundation, Cleveland, $\mathrm{OH} ;{ }^{3}$ University Hospitals of Cleveland, Cleveland, $\mathrm{OH} ;{ }^{4}$ National Institute of Diabetes and Digestive and Kidney Diseases, National Institutes of Health, Bethesda, MD; Martin Luther King-Drew Medical Center, Los Angeles, $C A ;^{6}$ and the University of Michigan Medical Center, Ann Arbor, $\mathrm{MI}^{7}$ Address for correspondence:

Robert A. Phillips, MD, PhD, Chairman, Department of Medicine, Lenox Hill Hospital, 100 East 77th

Street, New York, NY 10021

E-mail:rphillips@lenoxhill.net

Manuscript received February 18, 2004;

revised April 20, 2004;

accepted April 27, 2004

ID: 3555 insufficiency, also provided an opportunity to evaluate recruitment methods in minority populations. Eighty-three percent of patients were recruited through screening in clinical practice. To randomize 635 patients, 558,295 charts were reviewed (approximately 879 charts per randomized patient). More than half of the randomized patients ( $n=635$ or $58 \%$ ) were found by chart review. Sixty percent of women with creatinine levels considered within the normal range had at least mild chronic renal insufficiency. Screening in clinical practice was the most effective strategy to recruit participants with mild to moderate chronic renal insufficiency and bypertension into the clinical trial. This technique may also be an effective approach in trials of other essentially asymptomatic conditions. (J Clin Hypertens. 2004;6:430436) ${ }^{\circ} 2004$ Le Jacq Communications, Inc.

$\mathrm{E}$ nd-stage renal disease (ESRD) is a major health problem in the United States. In 1998 the number of incident and prevalent patients with ESRD exceeded 300,000 and has been steadily increasing. ${ }^{1}$ The increasing incidence of ESRD is in stark contrast to the significant decline in the death rates for cardiovascular disease and stroke observed during the past 20 years. African Americans bear a disproportionate burden of ESRD, ${ }^{1,2}$ in that they comprise about $13 \%$ of the US population ${ }^{3}$ but make up more than $40 \%$ of ESRD patients. ${ }^{1}$ The reasons for this discrepancy are not fully 
understood. Hypertension is the second leading cause of ESRD among African Americans, accounting for more than $30 \%$ of African-American patients receiving hemodialysis. ${ }^{1}$ Accordingly, high rates of hypertension, earlier age of occurrence, greater severity, and greater difficulty in controlling blood pressure among African Americans have all been regarded as possible factors to account for the higher prevalence of ESRD among this group. To identify approaches to prevent or delay the occurrence of ESRD among African Americans with hypertension, the National Institute of Diabetes and Digestive and Kidney Diseases conducted the African American Study of Kidney Disease and Hypertension (AASK), a randomized, controlled clinical trial evaluating two levels of blood pressure control and three antihypertensive drug regimens.

There is emerging literature on the recruitment of African Americans to clinical trials, but there is limited experience in recruitment of this minority segment of the US population into clinical trials of chronic renal disease. ${ }^{4-10}$ Consequently, the optimal strategies for recruitment of African Americans into renal clinical trials have not been identified. Despite having conducted a pilot study to evaluate recruitment strategies, ${ }^{10}$ it was anticipated that recruiting patients into the AASK fullscale trial would be difficult given the low awareness of hypertension, ${ }^{11}$ the asymptomatic nature of the early stages of chronic renal disease, and the skepticism among this group toward participation in clinical trials. ${ }^{12}$ We describe the recruitment techniques employed and their yield of randomized participants in the AASK full-scale trial.

\section{METHODS}

\section{Trial Design}

Details of the study design, including complete eligibility and exclusion criteria, have been described previously. ${ }^{13}$ Briefly, the randomized phase of AASK was a 21-center clinical trial aimed to compare the effect of two levels of blood pressure control and three antihypertensive drug regimens on the rate of decline in renal function among African Americans with hypertension-related renal insufficiency. Eligible patients were randomized in a $3 \times$ 2 factorial design to one of three antihypertensive drug regimens ( $\beta$ blocker, calcium channel blocker, or angiotensin-converting enzyme inhibitor) as initial therapy (initial therapy was double masked) and to a blood pressure control group (mean arterial pressure $\leq 92 \mathrm{~mm} \mathrm{Hg}$ or $102-107 \mathrm{~mm} \mathrm{Hg}$ ).

The study protocol was approved by the institutional review boards of the participating institutions
Table I. Centers Participating in the African American Study of Kidney Disease and Hypertension Trial

Alabama: University of Alabama, Birmingham

California: Harbor-UCLA Medical Center, Torrance; King-

Drew University, Los Angeles; University of Southern

California, Los Angeles; University of California, San Diego

District of Columbia: Howard University Hospital

Florida: University of Florida, Gainesville; University of Miami, Coral Gables

Georgia: Emory University, Decatur; Morehouse School of Medicine, Atlanta

Illinois: Rush-Presbyterian-St. Luke’s Medical Center, Chicago

Maryland: The Johns Hopkins University, Baltimore

Michigan: University of Michigan, Ann Arbor

New York: Harlem Hospital Center, New York; Mount Sinai Medical Center, New York

Ohio: Case Western Reserve University, Cleveland; Ohio State University, Columbus

South Carolina: Medical University of South Carolina, Charleston

Tennessee: Meharry Medical College, Nashville; Vanderbilt University, Nashville

Texas: University of Texas Southwestern Medical Center, Dallas

Table II. Randomization Goals by Clinical Center

\begin{tabular}{|lc|}
\hline CENTER & $\begin{array}{c}\text { RANDOMIZATION } \\
\text { GoAL (PoINTs) }\end{array}$ \\
\hline San Diego & 40 \\
Case Western Reserve University & 85 \\
Emory University & 50 \\
Harbor-UCLA & 24 \\
Howard University & 50 \\
Johns Hopkins University & 65 \\
King-Drew Medical Center & 39 \\
Medical University of South Carolina & 75 \\
Meharry Medical College & 20 \\
Harlem Hospital Medical Center & 20 \\
Morehouse School of Medicine & 20 \\
Mount Sinai Medical Center & 60 \\
Ohio State University & 80 \\
Rush-Presbyterian-St. Lukes Medical & 80 \\
Center & \\
University of Alabama at Birmingham & 65 \\
University of Florida at Gainesville & 65 \\
University of Miami & 75 \\
University of Michigan & 85 \\
University of Southern California & 43 \\
University of Texas Southwestern & 75 \\
Medical Center & 60 \\
Vanderbilt University & \\
\hline
\end{tabular}




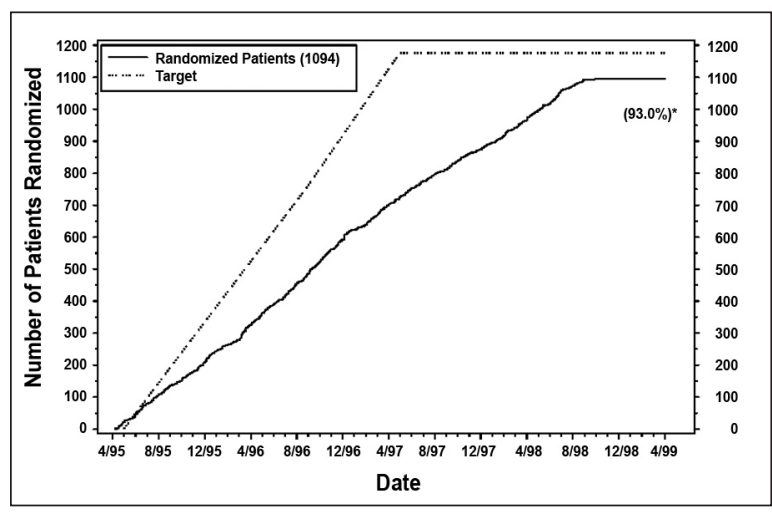

Figure 1. Time-course of recruitment (actual vs. target) in the African American Study of Kidney Disease and Hypertension. "Percent of goal

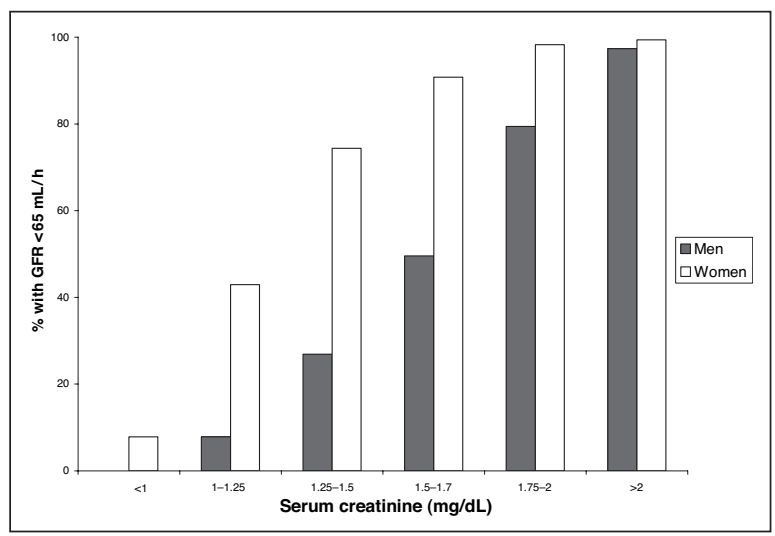

Figure 2. The relationship between the proportion of subjects with glomerular filtration rate (GFR) within study eligibility range and serum creatinine

and written informed consent was obtained in all subjects before enrollment in the trial. Location of clinical centers is listed in Table I.

\section{Recruitment Goals and Strategies}

The goal was to enroll 1176 patients. The randomization goals for the 21 clinical centers are listed in Table II. Based on the results of the AASK pilot study ${ }^{10}$ it was envisioned that most clinical centers would target their recruitment efforts at screening of patients in clinical practice. In addition, community-based approaches were implemented. The former strategy consisted of preidentification of prospective participants through review of hospital charts, review of computerized laboratory and demographic data, and referrals from primary care providers. In most cases, subjects targeted for further follow-up were identified by their serum creatinine value $(1.1-4.0 \mathrm{mg} / \mathrm{dL}$ in women and $1.3-4.5 \mathrm{mg} / \mathrm{dL}$ in men). Community-based recruitment strategies included mass mail, mass media (television and radio), screening at health fairs and churches, and referrals of relatives and friends of ESRD patients.

Typically, mass mail campaigns relied on targeted mailing of brochures or letters that described the trial and encouraged the recipient to contact a local clinical center for additional information or to schedule an appointment. Approaches using mass media were targeted to the African-American community when possible, and included radio and television interviews, newspaper articles, public service announcements on television, and paid advertisements in print media.

Community screenings that focused on blood pressure measurement (and occasionally included blood taken for a serum creatinine measurement) were conducted at health fairs, churches, shopping malls, pharmacies, work sites, and a variety of other settings. Recruitment materials were also distributed in these settings.

Recruitment of relatives or friends of ESRD patients was conducted in conjunction with patients' nephrologists. Typically, ESRD patients provided contact information that made it possible for recruitment staff at the local AASK clinical center to contact the relatives or friends by telephone or mail.

\section{Statistical Analyses}

Statistical analyses were performed using statistical packages by SAS Institute (Cary, NC) and SPSS, Inc. (Chicago, IL). Differences of $p<0.05$ were considered statistically significant.

\section{RESULTS}

\section{Timing of Recruitment}

Overall, 2801 subjects were screened during a 42-month period. The yield of randomized participants $(\mathrm{n}=1094)$ from the second screening visit was $39 \%$. To achieve the recruitment goals, the recruitment period was extended by 1 year (only approximately $800[68 \%$ ] subjects were recruited by September 1997). Overall, 1094 (93\% of planned) patients were recruited at the participating centers by the end of the planned recruitment period. The time course of the recruitment of study participants is shown in Figure 1.

\section{Baseline Clinical and Demographic Characteristics} Selected demographic and clinical characteristics of the randomized participants at baseline are shown in Table III. On average, randomized subjects were middle aged, overweight, and of low socioeconomic status. There were significantly more men than women in the study. By design, all of the subjects had hypertension; more than half had at least one comorbid 
condition in addition to hypertension. The majority of the randomized subjects were not married. More than $40 \%$ had not finished high school, 37\% were employed, approximately half of the subjects had a household income $<\$ 15,000$ per year.

\section{Yield of Randomized Participants}

Eighty-three percent of randomized patients were recruited through screening in clinical practice. Nearly three quarters were contacted directly by an AASK team member, the remainder were referred by other health professionals (Table IV). There was general agreement with the observations of the pilot study in that chart or laboratory record review was the major source of randomized participants. A total of 558,295 medical charts or laboratory reports were examined resulting in 635 randomized participants. Thus, on average 879 medical records were reviewed for each randomized participant. The number of medical records or laboratory reports reviewed to yield one randomized participant varied significantly across clinical centers, from 42 to 6381 .

Recruitment in clinical practice and by mass mail were the most efficient strategies with yields (number randomized/number screened) of $42 \%$ and $39 \%$, respectively. Other recruitment approaches had a yield of $26 \%$. This probably resulted from the difference in serum creatinine level in patients recruited by different strategies: $1.8 \pm 1.0 \mathrm{mg} / \mathrm{dL}$ in patients recruited from clinical practice vs. $1.9 \pm 0.9$ $\mathrm{mg} / \mathrm{dL}$ in patients recruited through mass mailing ( $p=$ nonsignificant vs. patients recruited from clinical practice) and $1.6 \pm 1.0 \mathrm{mg} / \mathrm{dL}$ in patients recruited through other strategies $(p<0.01$ vs. the other two groups). It should be noted that the number of patients recruited via mass mailing was small (52 subjects) and the majority $(60 \%)$ of patients recruited were from one site. Thus, the generalizability regarding the efficacy of this strategy may be limited.

\section{Reasons for Exclusion From the Study}

Overall, 1036 men and 672 women $(60 \%$ of the screened men and $61 \%$ women) were excluded. The major reason for exclusion before measurement of glomerular filtration rate (GFR) was patients' serum creatinine level that the study team considered either too low or high. After the GFR measurements, the main reason was GFR out of range of eligibility for the study. Women were significantly more likely to be excluded from the study on the basis of serum creatinine level than men $(32 \%$ vs. $23 \% ; p<0.001)$. This prob-

\begin{tabular}{|c|c|}
\hline \multicolumn{2}{|c|}{$\begin{array}{l}\text { Table III. Selected Clinical and Demographics at Baseline } \\
\text { of the Randomized Study Participants }\end{array}$} \\
\hline Age (years $\pm S D)$ & $54.5 \pm 10.7$ \\
\hline \multicolumn{2}{|l|}{ Gender (n [\%]) } \\
\hline Males & $669(61)$ \\
\hline Females & $425(39)$ \\
\hline $\mathrm{BMI}($ mean $\pm \mathrm{SD})$ & $30.6 \pm 6.6$ \\
\hline Systolic BP (mm Hg \pm SD) & $150 \pm 24$ \\
\hline Diastolic BP $(\mathrm{mm} \mathrm{Hg} \pm \mathrm{SD})$ & $96 \pm 14$ \\
\hline Serum creatinine $(\mathrm{mg} / \mathrm{dL} \pm \mathrm{SD})$ & $2.0 \pm 0.7$ \\
\hline GFR $\left(\mathrm{mL} / \mathrm{min} / 1.73 \mathrm{~m}^{2} \pm \mathrm{SD}\right)$ & $46 \pm 13$ \\
\hline Years with hypertension $( \pm$ SD) & $14.2 \pm 10.1$ \\
\hline Number of antihypertensives $( \pm S D)$ & $2.5 \pm 1.1$ \\
\hline \multicolumn{2}{|l|}{ Comorbid conditions (n [\%]) } \\
\hline None & $527(48)$ \\
\hline One & $420(38)$ \\
\hline Two or more & $147(13)$ \\
\hline \multicolumn{2}{|l|}{ Marital status (n [\%]) } \\
\hline Never married & $227(21)$ \\
\hline Married/married-like & $394(36)$ \\
\hline Divorced/separated & $338(31)$ \\
\hline Widow(er) & $134(12)$ \\
\hline No information & $1(0.1)$ \\
\hline \multicolumn{2}{|l|}{ Highest education level (n [\%]) } \\
\hline Below high school & $444(41)$ \\
\hline High school & $326(30)$ \\
\hline College or above & $322(29)$ \\
\hline No information & $2(0.2)$ \\
\hline \multicolumn{2}{|l|}{ Employment status (n [\%]) } \\
\hline Employed & $404(37)$ \\
\hline Unemployed & $308(28)$ \\
\hline Retired & $277(25)$ \\
\hline Homemaker & $37(3)$ \\
\hline Other/no information & $68(6)$ \\
\hline \multicolumn{2}{|l|}{ Annual household income (n [\%]) } \\
\hline$<\$ 15,000$ & $521(48)$ \\
\hline$\$ 15,000-\$ 39,000$ & $280(26)$ \\
\hline$>\$ 40,000$ & $90(8)$ \\
\hline Declined to provide info & $203(19)$ \\
\hline
\end{tabular}

ably resulted in a lower rate of exclusion because of the GFR not within the range of eligibility ( $33 \%$ vs. $45 \% ; p<0.001)$.

\section{Relationship Between Creatinine Value and Level of Renal Function}

Although women had significantly lower mean serum creatinine levels than men they had lower mean GFRs (Table V). A significant proportion of subjects with serum creatinine levels in the normal 


\begin{tabular}{|lcc|}
\hline \multicolumn{2}{|l|}{ Table IV. Sources of Subject Recruitment } \\
\hline \multicolumn{2}{|c|}{$\mathrm{N}$} & $\%$ \\
\hline Screening in clinical practice & 703 & 64 \\
AASK team member & 212 & 19 \\
Other health professional & & \\
Community recruiting & 52 & 5 \\
Mass mail & 57 & 5 \\
Mass media & 28 & 3 \\
Relatives/friends & 3 & 0.3 \\
Community screening & 39 & 4 \\
Other/unknown & \multicolumn{2}{l}{} \\
\hline AASK=African American Study of Kidney Disease and \\
Hypertension study
\end{tabular}

range had renal insufficiency (Figure 2). Women with serum creatinine levels within normal range were significantly more likely than men to have renal insufficiency $(60 \%$ of women and $23 \%$ of men with serum creatinine level between $1 \mathrm{mg} / \mathrm{dL}$ and $1.5 \mathrm{mg} / \mathrm{dL} ; p<0.001)$.

\section{DISCUSSION}

AASK is the largest clinical trial of chronic renal disease that consisted exclusively of African Americans. The goal for randomization was essentially achieved but required extending the recruitment period by 12 months. This goal was attained despite the skepticism among African Americans regarding clinical trials, ${ }^{12}$ the relatively narrow range of renal function eligibility, and the largely asymptomatic nature of mild to moderate chronic renal insufficiency.

The main results of the AASK trial have been previously reported. ${ }^{14}$ After recruitment and randomization, patients were followed up for 3-6.4 years. Changes in GFR between randomized groups was the primary trial outcome in AASK. Prespecified secondary analyses focused on clinical events (a composite outcome defined by the occurrence of ESRD, reducing GFR by $50 \%$, or death), an outcome that is of direct relevance to practicing clinicians. Randomized, double-blind comparisons of the antihypertensive groups were inconclusive for the primary outcome of mean change in GFR, but demonstrated that ramipril, compared with amlodipine and metoprolol, more effectively reduced clinical events in patients with hypertensive renal disease. Furthermore, metoprolol appeared to be superior to amlodipine in reducing ESRD or death. In these clinical outcome analyses, the risk reduction in clinical end points for the ramipril group was not significantly related to the level of proteinuria at baseline, but patients in the subgroup with urinary protein/creatinine ratio $>0.22$ were more likely to have an event than those in the subgroup with urinary protein/creatinine ratio $<0.22$.

After recruitment, patients were also randomized to two blood pressure goals: a usual blood pressure goal of mean arterial pressure (MAP) between $102 \mathrm{~mm} \mathrm{Hg}$ and $107 \mathrm{~mm} \mathrm{Hg}$, or a low MAP goal of $<92 \mathrm{~mm} \mathrm{Hg}$. Despite a mean MAP separation of approximately $10 \mathrm{~mm} \mathrm{Hg}$ throughout the study, neither the mean change in GFR or the rates of clinical end points differed significantly between the low blood pressure group (average blood pressure of $128 / 78 \mathrm{~mm} \mathrm{Hg}$ ) and the usual blood pressure group (average blood pressure of $141 / 85 \mathrm{~mm} \mathrm{Hg}$ ).

Several of the demographic and clinical characteristics of the recruited population are noteworthy because they may have influenced recruitment, especially the low level of education and income. On average, the subjects in AASK were of lower socioeconomic class and more likely to be men. The former observation is consistent with the finding that lower socioeconomic status is independently associated with renal disease. ${ }^{2}$ Some data on the relationship between demographic characteristics and success rate of patient recruitment suggest that male and less-educated subjects are more likely to drop out of a study during the screening phase, ${ }^{15}$ but this was not the experience in our trial. Patient preference was an uncommon reason for exclusion from the trial, accounting for $<15 \%$ of excluded subjects. Several factors can account for the recruitment of significantly more men then women in the study: renal dysfunction is more common in men than in women, ${ }^{16}$ and there may have been a bias in the screening process. The most common reason for exclusion from the study during the screening phase was serum creatinine level that made the study team not proceed with GFR measurements. Because women have lower creatinine levels for a given GFR (Table V, Figure 2, and Coggins et al. ${ }^{17}$ ), some women with GFRs within

\begin{tabular}{|lccc|}
\hline \multicolumn{4}{|c|}{ Table V. Serum Creatinine Levels and Renal Function in Men and Women in the Study } \\
\hline \\
\hline Serum creatinine $(\mathrm{mg} / \mathrm{dL} \pm \mathrm{SD})$ & Women $(\mathrm{N}=425)$ & $P$ VALUE \\
Glomerular filtration rate & $2.18 \pm 0.76$ & $1.77 \pm 0.57$ & $<0.001$ \\
$\left(\mathrm{~mL} / \mathrm{min} / 1.73 \mathrm{~m}^{2} \pm \mathrm{SD}\right)$ & $46.9 \pm 13.1$ & $43.7 \pm 12.6$ & $<0.001$ \\
\hline
\end{tabular}


the range of eligibility for the study may have been excluded before the GFR measurements. The finding that women in the study had lower GFRs than men further supports this possibility.

We found that a significant proportion of subjects with serum creatinine levels within a range traditionally considered normal had at least mild renal dysfunction. This was significantly more common among women than men. Thus, a substantial proportion of African-American women with mild to moderate renal insufficiency are not identified by standard tests of renal function. This finding has important public health implications because the blood pressure goals in treatment of hypertension are lower in subjects with renal disease. ${ }^{18}$ Lowering of the upper limit of normal creatinine values to $1.2 \mathrm{mg} / \mathrm{dL}$ in women will identify more women with renal disease. At this level, a middle-aged, African-American women with hypertension will have a $50 \%$ chance of having renal insufficiency.

The AASK pilot study demonstrated that it was feasible to recruit subjects into the trial and provided valuable information on the comparative value of different approaches to recruitment of subjects from within this population. The pilot was very predictive, but at the same time it was also very misleading. Screening of prospective participants in clinical practice yielded the most randomized patients, as predicted by the pilot. However, it required significantly more effort in the full-scale trial than in the pilot. In contrast to the pilot study, where review of approximately 10 charts yielded a randomized patient, it was a much less effective strategy in the full-scale trial. The number of charts or laboratory reports reviewed to yield a randomized patient varied 150-fold across the sites (from 42 to 6381), with the average of 879 charts or laboratory reports reviewed per randomized patient. This unanticipated difficulty was the major reason for the extension of the recruitment period. The reasons for the differences in the number of charts reviewed per randomized patient are likely to be the sources of medical records at different sites. These sources varied from the lists of patients in public health clinics to computerized searches based on serum creatinine values.

Although there was a substantial variability among the participating centers in recruitment strategies utilized as well as their relative efficacy, community recruitment was not an efficient strategy both in the pilot and in the full-scale trial. This is consistent with the recruitment experience in the Modification of Diet in Renal Disease
(MDRD) study. ${ }^{9}$ One of the reasons for that may be low awareness of essentially asymptomatic diseases such as hypertension and renal dysfunction in the community. Indeed, the National Health and Nutrition Examination Survey (NHANES) demonstrated an insufficient awareness of hypertension in the US population. ${ }^{11}$ Similar rates of awareness were demonstrated among African Americans. ${ }^{19,20}$ Thus, having the subjects' medical information screened either by the AASK team members through chart review or by their primary physicians provided more patients than community recruitment, which relies on the subjects' awareness of their medical conditions.

Community recruitment strategies were utilized with good results in several hypertension trials, ${ }^{15,21-25}$ but to be effective, the community recruitment required a colossal effort. For example, in the Systolic Hypertension in the Elderly Program (SHEP), more than 3.4 million letters of invitation were mailed with a response rate of around $4 \% .^{25}$ In the Dietary Approach to Stop Hypertension (DASH) trial, ${ }^{24} 347,500$ brochures were mailed, 250,500 coupons were distributed, 114 advertisements were published, 214 radio and television advertisements were broadcast, and 68 screening events and presentations were conducted to enroll a total of 459 participants. The enormous effort involved in community recruitment required several innovative approaches to increase the cost-effectiveness of this strategy. These include use of community volunteers to decrease the costs of the recruitment ${ }^{26}$ as well as increasing the awareness of the study through a specialized community network. ${ }^{27}$

Difficulties in recruitment may be one reason for the inadequate representation of African Americans in clinical trials. Optimal strategies for recruitment of minority subjects have not been delineated and are likely to vary with the population and conditions studied. The recruitment experience in the AASK trial suggests that when the condition studied is essentially asymptomatic, screening in clinical practice, which includes review of charts and computerized laboratory and demographic data, may be the strategy generating the most randomized subjects. Comparative analyses of the experiences of other clinical trials are needed to establish the most efficient strategies for recruitment of minority participants. Finally, the impact of the Health Insurance Portability and Accountability Act regulations ${ }^{28}$ on the most successful recruiting strategies employed in AASK, and hence the ability to recruit African Americans into clinical trials, may be substantial. 
Acknowledgment and disclosures: A list of the African American Study of Kidney Disease and Hypertension (AASK) study group participants can be obtained from Dr. Phillips. In addition to funding under a cooperative agreement from NIDDK, this study was supported in part by the following institutional GCRC and other NIH grants: NIH, M01 RR-00080, 5M01 RR-00071, M01 00032, P20-RR11145, M01 RR00827, M01 RR00052, 2P20 RR11104, DK 2818-02, RR11145 (NIH RCMI/NCRR) and DK45426-04 (NIH/NIDDK). Support was also received from the Office of Research in Minority Health and drugs were donated and some financial support provided to NIDDK by Pfizer Inc., AstraZeneca Pharmaceuticals, and King Pharmaceuticals, Inc.

\section{REFERENCES}

1 US Renal Data System. 2000 Annual Data Report. Bethesda, MD: National Institute of Diabetes and Digestive and Kidney Diseases; 2001.

2 Klag MJ, Whelton PK, Randall BL, et al. End-stage renal disease in African-American and white men. 16-year MRFIT findings. JAMA. 1997;277:1293-1298.

3 US Census Bureau. Annual population estimates by sex, race and Hispanic origin, selected years from 1990 to 2000. Available at: http://eire.census.gov/popest/archives/ national/nation3/intfile3-1.txt. Accessed May 13, 2004.

4 Paskett ED, DeGraffinreid C, Tatum CM, et al. The recruitment of African-Americans to cancer prevention and control studies. Prev Med. 1996;25:547-553.

5 Coleman EA, Tyll L, LaCroix AZ, et al. Recruiting AfricanAmerican older adults for a community-based health promotion intervention: which strategies are effective? Am J Prev Med. 1997;13:51-56.

6 Mabunda-Temple G. Recruitment of African Americans in AIDS clinical trials: some recommended strategies. ABNF J. 1998;9:61-64.

7 Holcombe RF, Jacobson J, Li A, et al. Inclusion of black Americans in oncology clinical trials: the Louisiana State University Medical Center experience. Am J Clin Oncol. 1999;22:18-21.

8 Royal C, Baffoe-Bonnie A, Kittles R, et al. Recruitment experience in the first phase of the African American Hereditary Prostate Cancer (AAHPC) study. Ann Epidemiol. 2000;10: S68-S77.

9 Kusek JW, Coyne T, de Velasco A, et al. Recruitment experience in the full-scale phase of the Modification of Diet in Renal Disease Study. Control Clin Trials. 1993;14:538-557.

10 Whelton PK, Lee JY, Kusek JW, et al. Recruitment experience in the African American Study of Kidney Disease and Hypertension (AASK) pilot study. Control Clin Trials. 1996;17:17S-33S.

11 Burt VL, Whelton P, Roccella EJ, et al. Prevalence of hypertension in the US adult population. Results from the Third National Health and Nutrition Examination Survey, 1988-1991. Hypertension. 1995;25:305-313.

12 Corbie-Smith G, Thomas SB, Williams MV, et al. Attitudes and beliefs of African Americans toward participation in medical research. J Gen Intern Med. 1999;14:537-546.
13 Agodoa L. African American Study of Kidney Disease and hypertension (AASK) - clinical trial update. Ethn Dis. 1998;8:249-253.

14 Wright JT Jr, Bakris G, Greene T, et al. Effect of blood pressure lowering and antihypertensive drug class on progression of hypertensive kidney disease: results from the AASK trial. JAMA. 2002;288:2421-2431.

15 Hollis JF, Satterfield S, Smith F, et al. Recruitment for phase II of the Trials of Hypertension Prevention. Effective strategies and predictors of randomization. Trials of Hypertension Prevention (TOHP) Collaborative Research Group. Ann Epidemiol. 1995;5:140-148.

16 Jungers P, Chauveau P, Descamps-Latscha B, et al. Age and gender-related incidence of chronic renal failure in a French urban area: a prospective epidemiologic study. Nephrol Dial Transplant. 1996;11:1542-1546.

17 Coggins $\mathrm{CH}$, Breyer LJ, Caggiula AW, et al. Differences between women and men with chronic renal disease. Nephrol Dial Transplant. 1998;13:1430-1437.

18 The seventh report of the Joint National Committee on prevention, detection, evaluation, and treatment of high blood pressure; the JNC 7 report. JAMA. 2003;289:2560-2572.

19 Bone LR, Hill MN, Stallings R, et al. Community health survey in an urban African-American neighborhood: distribution and correlates of elevated blood pressure. Ethn Dis. 2000:10:87-95.

20 Pavlik VN, Hyman DJ, Vallbona C, et al. Hypertension awareness and control in an inner-city African-American sample. J Hum Hypertens. 1997;11:277-283.

21 Ford CE, Langford HG, Palmer MJ. Recruitment in the hypertension detection and follow-up program. Control Clin Trials. 1987;8:54S-67S.

22 Whelton PK, Babnson J, Appel LJ, et al. Recruitment in the Trial of Nonpharmacologic Intervention in the Elderly (TONE). I Am Geriatr Soc. 1997;45:185-193.

23 Vollmer WM, Svetkey LP, Appel LJ, et al. Recruitment and retention of minority participants in the DASH controlled feeding trial. DASH Collaborative Research Group. Dietary Approaches to Stop Hypertension. Ethn Dis. 1998;8:198-208.

24 Appel LJ, Vollmer WM, Obarzanek E, et al. Recruitment and baseline characteristics of participants in the Dietary Approaches to Stop Hypertension trial. DASH Collaborative Research Group. J Am Diet Assoc. 1999;99:S69-S75.

25 Cosgrove N, Borhani NO, Bailey G, et al. Mass mailing and staff experience in a total recruitment program for a clinical trial: the SHEP experience. Systolic Hypertension in the Elderly Program. Cooperative Research Group. Control Clin Trials. 1999;20:133-148.

26 Hertert S, Bailey G, Cottinghan V, et al. Community volunteers as recruitment staff in a clinical trial: the systolic hypertension in the elderly program (SHEP) experience. Control Clin Trials. 1996;17:23-32.

27 Gorelick PB, Richardson D, Hudson E, et al. Establishing a community network for recruitment of African Americans into a clinical trial. The African-American antiplatelet stroke Prevention Study (AAASPS) experience. J Natl Med Assoc. 1996;88:701-704.

28 Health Insurance Portability and Accountability Act (HIPPA). Available at: http//www.cms.gov/hippa. Accessed July 7, 2004. 